\title{
ESTUDO IN SILICO DE COMPLEXOS DE INCLUSÃO COM CICLODEXTRINAS PARA INCREMENTO DE SOLUBILIDADE DA CANTIN-6-ONA
}

Fabíola F. F. G. de Oliveira ${ }^{1}$; Marcelo M. Rabello²; Larissa A. Rolim²; Marcelo Z. Hernandes $^{3}$; Pedro J. Rolim Neto ${ }^{3}$

${ }^{1}$ Centro Universitário Estácio do Recife, Campus Abdias de Carvalho.

2 Universidade Federal do Vale de São Francisco, Central de Análise de Fármacos, Medicamentos e Alimentos.

${ }^{3}$ Universidade Federal de Pernambuco, Centro de Ciências da Saúde, Departamento de Farmácia.

E-mail para contato: montenegro.rabello@gmail.com

RESUMO - Foi realizado um estudo de modelagem molecular com o objetivo de compreender a formação dos complexos de inclusão entre os hosts $\beta$ ciclodextrina ( $\beta-C D)$ e hidroxipropil- $\beta-C D(H P-\beta-C D)$ e o guest Cantin-6-ona. Para construir o modelo do derivado $H P-\beta-C D$ foram geradas um total de 1000 estruturas (40 configurações com 25 conformações diferentes, cada). Para a realização dos cálculos de docking molecular foi utilizado o programa Autodock VINA. Toda a metodologia de modelagem molecular foi realizada de forma automatizada, utilizando a plataforma CycloMolder. Os resultados de docking apresentaram duas orientações principais em relação a posição da molécula guest (Cantin-6-ona) incluída na molécula host. A primeira, chamada de orientação I, apresenta a porção naftiridina voltada para a borda mais larga do host, e a outra, orientação II, com a porção indol voltada para a borda mais larga. A melhor solução de docking para o complexo $\beta$-CD:Cantin-6-ona apresentou energia de -5,3 kcal/mol. Já para o complexo HP- $\beta$-CD:Cantin-6-ona, a melhor solução da orientação I apresentou energia de $-6,5 \mathrm{kcal} / \mathrm{mol}$, enquanto para a orientação II a energia foi de -6,1 kcal/mol. Os estudos da modelagem molecular finais apontam o complexo HP- $\beta$-CD:Cantin-6-ona $(-6,5$ e -6,1 kcal $/ \mathrm{mol})$ como mais estável que o complexo $\beta$-CD:Cantin-6-ona $(-5,3 \mathrm{kcal} / \mathrm{mol})$, portanto acredita-se que também seja mais eficiente no incremento da solubilidade em água da Cantin-6-ona.

Palavras chave: Cantin-6-ona. Ciclodextrina. Modelagem molecular.

ABSTRACT - A molecular modeling study was carried out to understand the formation of inclusion complexes between $\beta$-cyclodextrin $(\beta-C D)$ and hydroxypropyl- $\beta-C D$ (HP- $\beta-C D)$ hosts and the guest Cantin-6-one. To build the model of HP- $\beta-C D$ derivative, a total of 1000 structures (40 configurations with 25 different conformations, each) were generated. The Autodock VINA program was used to perform the molecular docking calculations. All the molecular modeling methodology was performed in an automated fashion, using the 
CycloMolder platform. The docking results showed two main orientations regarding the position of the guest molecule (Canthin-6-one) included in the host molecule. The first known as orientation I, has the naphthyridine moiety at the wider rim of the host, and the other, known as orientation II, has the indole moiety at the wider rim. The best docking solution for the $\beta$-CD:Canthin-6-one complex had energy of $-5.3 \mathrm{kcal} / \mathrm{mol}$. For the HP- $\beta$-CD:Canthin-6-one complex, the best solution of orientation I had energy of $-6.5 \mathrm{kcal} / \mathrm{mol}$, whereas for orientation II the energy was $-6.1 \mathrm{kcal} / \mathrm{mol}$. The final molecular modeling studies point to the $H P-\beta$-CD:Cantin-6-one complex (-6.5 and $-6.1 \mathrm{kcal} / \mathrm{mol})$ as more stable than the $\beta$-CD:Cantin-6-one complex $(-5.3 \mathrm{kcal} / \mathrm{mol})$, so it is believed to be also more efficient in increasing the water solubility of Cantin-6-one.

Keywords: Canthin-6-one. Cyclodextrin. Molecular modelling.

\section{INTRODUÇÃO}

As ciclodextrinas (CDs) são oligossacarídeos cíclicos, cujas estruturas são formadas por unidades de glicopiranose. Devido à conformação em cadeira das unidades de glicopiranose, as CDs possuem a forma de um tronco de cone, com grupos hidroxila primários na borda estreita, e grupos hidroxila secundários que se prolongam a partir da extremidade mais larga (KURKOV; LOFTSSON, 2013).

As orientações individuais das unidades de glicopiranose apresentam grupos hidroxilas em ambas as bordas do cone, o que tornam as CDs solúveis em água. O interior da cavidade, no entanto, tem caráter hidrofóbico, fornece uma matriz hidrofóbica que permite a formação de complexos de inclusão com moléculas apolares ou pouco polares (SAENGER et al., 1998).

Um complexo de inclusão é formado por um host (hospedeiro), e por um guest (hóspede). Nesse caso, o host seria a CD (ou seu derivado) e o guest seria um fármaco (ou uma molécula bioativa), formando o complexo host-guest (LOFTSSON; DUCHÊNE, 2007). Os estudos de modelagem molecular estão presentes na maioria dos trabalhos envolvendo as CDs ou seus derivados, normalmente com o objetivo de elucidar as interações intermoleculares que estabilizam a formação do complexo host-guest (SILVA et al., 2016; XAVIER-JUNIOR et al., 2017).

A Cantin-6-ona é um alcaloide $\beta$-carbolina isolado de várias fontes de plantas, principalmente das famílias Rutaceae e Simaroubaceae, e recentemente de fungos (DAI et al., 2016). A Cantin-6-ona foi primeiramente isolada em 1952 da árvore australiana Pentaceras australis (HAYNES; NELSON; PRICE, 1952). Este composto apresenta uma variedade de efeitos farmacológicos, tais como atividades antifúngicas, antibacterianas, anticancerígenas e antivirais. (TREMMEL et al., 2016; VIEIRA TORQUATO et al., 2017).

A Cantin-6-ona apresenta uma estrutura plana, devido à presença de anéis aromáticos conjugados, como pode ser visto na Figura 1. O seu uso terapêutico está limitado principalmente devido a sua baixa solubilidade em água (DAI et al., 2016). A modelagem molecular (modelos in silico) possibilita calcular a energia de interação entre complexos de inclusão e elucidar as razões intermoleculares que justifiquem a formação destes. Portanto, este trabalho tem o objetivo de avaliar a formação dos complexos de inclusão com os hosts $\beta$-CD e HP- $\beta$-CD para o incremento da solubilidade da Cantin-6ona, através da aplicação de modelos in silico. 
Figura 1 - Estrutura tridimensional da Cantin-6-ona.

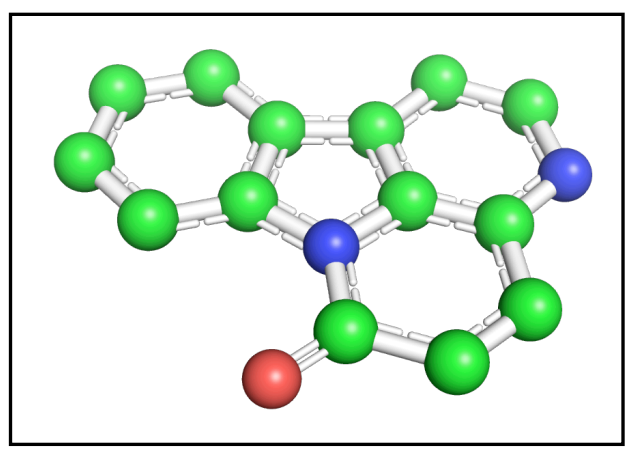

\section{MATERIAIS E MÉTODOS}

Para levar em conta aspectos relevantes da síntese de derivados de $\mathrm{CD}$, os seguintes pontos foram considerados: (i) A regiosseletividade da reação ocorre preferencialmente com as hidroxilas primárias $\mathrm{OH}$ (6), uma vez que são mais acessíveis, seguido das hidroxilas secundárias $\mathrm{OH}(2)$ com a maior acidez ( $p K a=12,2)$ (WENZ, 1994). (ii) a formação de derivados homólogos com os índices de substituição molar (MS) (TREIB et al., 1999) mais baixo e mais alto também são obtidos, além do produto (WENZ, 1994).

Nossa abordagem foi construir um modelo com 1000 estruturas (40 configurações com 25 conformações diferentes, cada) para o derivado HP- $\beta-C D$, a partir da estrutura tridimensional da $\beta$-CD (SAENGER et al., 1998). As 40 configurações foram construídas considerando os dois aspectos da síntese mencionados acima. Quanto à razão de MS de 0,7 parece razoável assumir que a estrutura da HP- $\beta$-CD ( 7 unidades de glicose) possui, em média, 5 unidades HP. Portanto, foram construídas 20 configurações com 5 unidades de HP, 10 com 4 unidades de HP e outras 10 com 6 unidades de HP.

Para seleção da posição de substituição de cada unidade de HP adicionada, durante a construção das estruturas, foi considerada a probabilidade de $70 \%$ para $\mathrm{OH}(6), 20 \%$ para $\mathrm{OH}(2)$ e $10 \%$ para $\mathrm{OH}$ (3). Os valores de energia de interação intermolecular para os complexos de inclusão host:guest foram calculados utilizando o programa de Autodock VINA (TROTT; OLSON, 2010), considerando toda a estrutura do host como sitio ativo e com o parâmetro exhaustiveness ajustado em 8 . Toda a metodologia de modelagem molecular foi realizada de forma automatizada, utilizando a plataforma CycloMolder (RABELLO, 2016).

\section{RESULTADOS E DISCUSSÃO}

Os resultados do estudo de modelagem molecular apontaram a formação de complexos de inclusão onde a molécula guest (Cantin-6-ona) se apresentou em duas orientações distintas. A primeira orientação com a porção naftiridina voltada para a borda mais larga da $\beta$-CD (Orientação I), e a segunda com a porção indol voltada para a borda larga (Orientação II).

Para o complexo $\beta$-CD:Cantin-6-ona a melhor solução de docking com energia de $-5,3 \mathrm{kcal} / \mathrm{mol}$ (Orientação I) pode ser observada na Figura 2. A solução é estabilizada por alguns contatos hidrofóbicos e por 2 ligações de hidrogênio (3,2 e 3,3 $\AA$ ). 
Figura 2 - Melhor solução de docking para o complexo $\beta$-CD:Cantin-6-ona, visão frontal (A) e lateral (B).

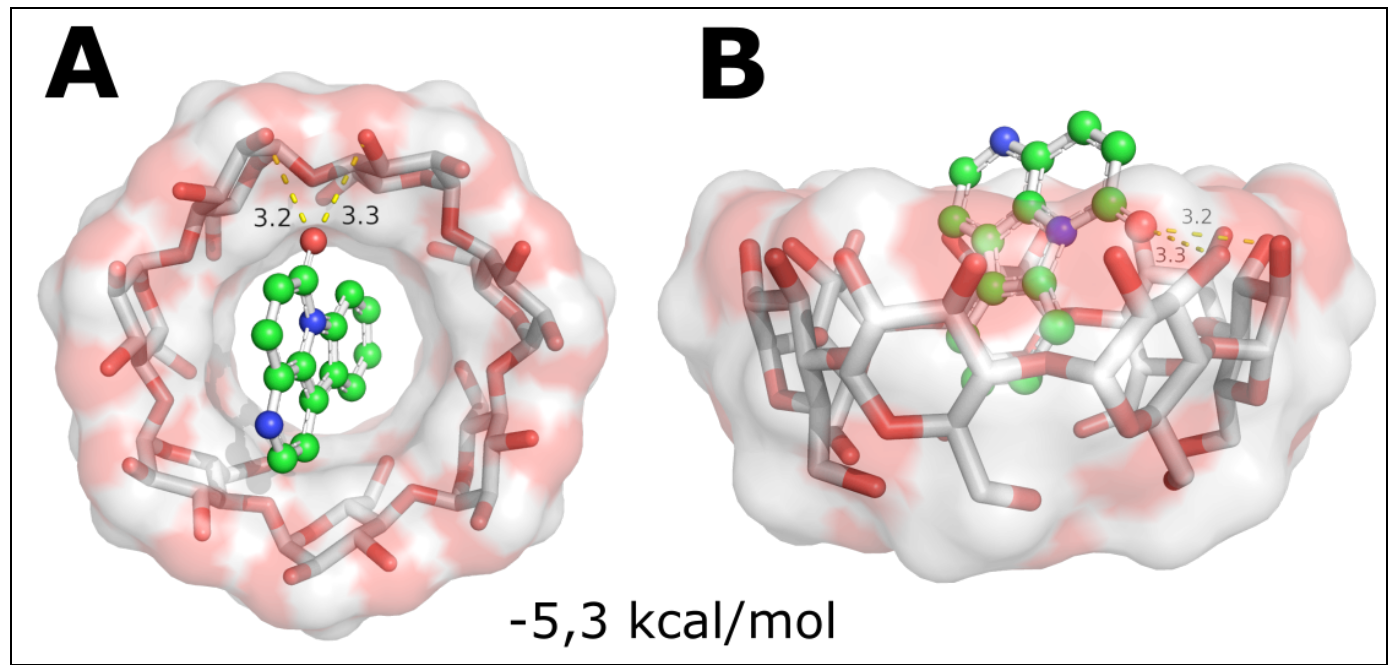

No estudo do complexo HP- $\beta$-CD:Cantin-6-ona a média energética para as 8 melhores soluções de docking, considerando a orientação I, é de $-6,4 \mathrm{kcal} / \mathrm{mol}$. Enquanto a média das 8 melhores soluções para a orientação II é de $-5,8 \mathrm{kcal} / \mathrm{mol}$, ver Tabela 1 . A melhor solução para a orientação I é mostrada na Figura 3A, com energia de $-6,5$ $\mathrm{kcal} / \mathrm{mol}$, enquanto a melhor solução de docking para a orientação II pode ser encontrada na Figura 3B, com energia de $-6,1 \mathrm{kcal} / \mathrm{mol}$. A melhor solução para a orientação I é estabilizada por vários contatos hidrofóbicos e por 2 ligações de hidrogênio (3,2 e 3,3 $\AA$ ), enquanto a melhor solução para a orientação II também é estabilizada por vários contatos hidrofóbicos e por 2 ligações de hidrogênio (3,0 e 3,3 $\AA$ ). Os resultados específicos para o complexo de inclusão HP- $\beta$-CD:Cantin-6-ona apontam a orientação I (-6,5 kcal $/ \mathrm{mol})$ como mais estável que a orientação II $(-6,1 \mathrm{kcal} / \mathrm{mol})$, devendo ocorrer em maior proporção.

Tabela 1 - Sumário das 8 melhores soluções de docking para o complexo HP- $\beta$ CD:Cantin-6-ona nas orientações I e II.

\begin{tabular}{|c|c|c|}
\hline Orientação I & $\mathrm{kcal} / \mathrm{mol}$ & Orientação II \\
\hline$-6,5$ & $\mathbf{1}^{\mathbf{o}}$ & $-6,1$ \\
\hline$-6,4$ & $\mathbf{2}^{\mathbf{o}}$ & $-5,9$ \\
\hline$-6,4$ & $\mathbf{3}^{\mathbf{o}}$ & $-5,8$ \\
\hline$-6,4$ & $\mathbf{4}^{\mathbf{o}}$ & $-5,8$ \\
\hline$-6,4$ & $\mathbf{5}^{\mathbf{0}}$ & $-5,7$ \\
\hline$-6,3$ & $\mathbf{6}^{\mathbf{0}}$ & $-5,7$ \\
\hline$-6,3$ & $\mathbf{7}^{\mathbf{0}}$ & $-5,6$ \\
\hline$-6,3$ & $\mathbf{8}^{\mathbf{0}}$ & $-5,4$ \\
\hline $\mathbf{- 6 , 4}$ & Média & $\mathbf{- 5 , 8}$ \\
\hline
\end{tabular}


Figura 3 - Melhores soluções de docking para o complexo HP- $\beta$-CD:Cantin-6-ona na orientação I (A) e orientação II (B).

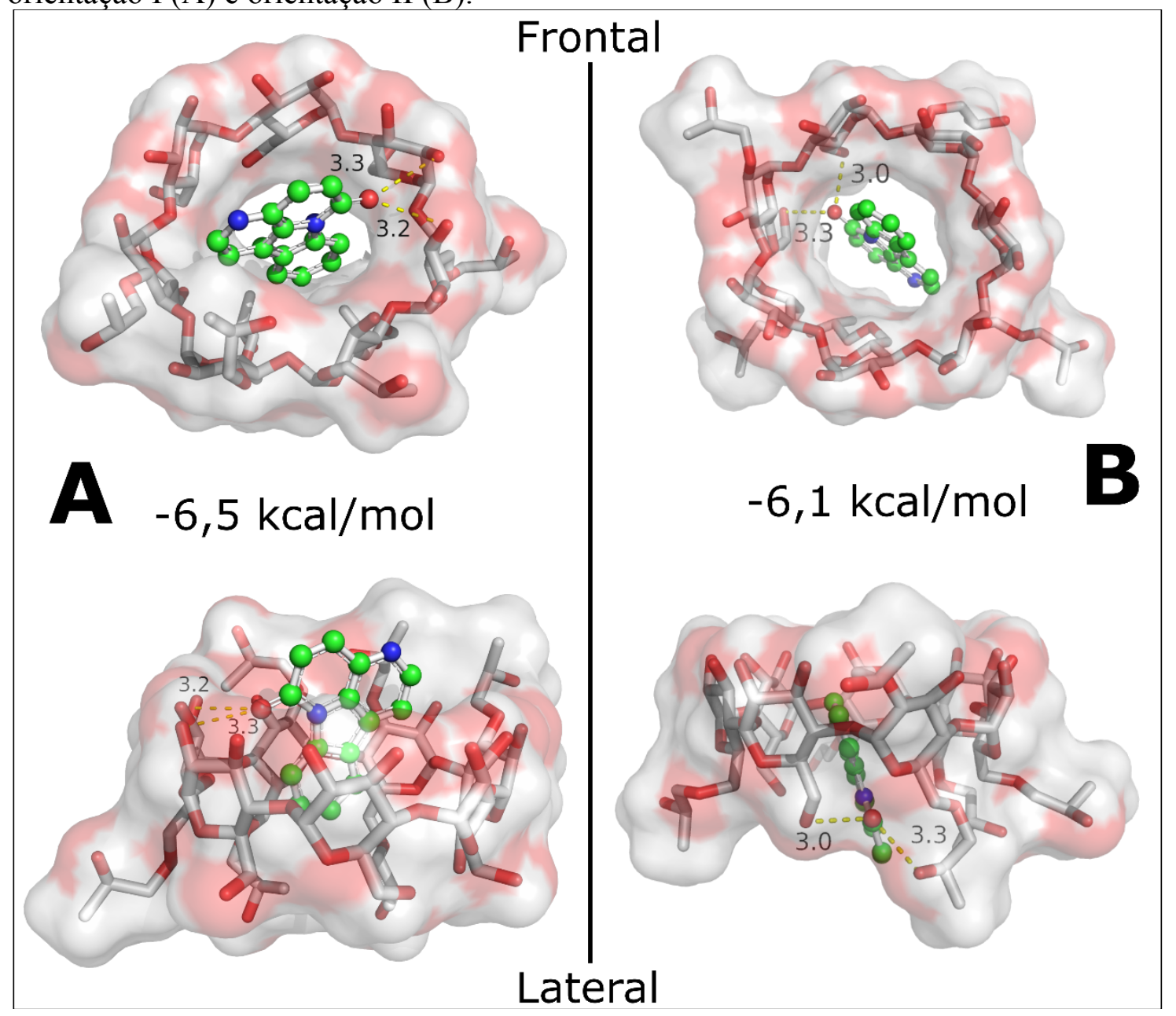

\section{CONCLUSÃO}

Os estudos da modelagem molecular finais apontam o complexo HP- $\beta$-CD:Cantin6-ona (-6,5 e -6,1 kcal/mol) como mais estável que o complexo $\beta$-CD:Cantin-6-ona (-5,3 $\mathrm{kcal} / \mathrm{mol}$ ). As interações, principalmente hidrofóbicas, adicionais entre a Cantin-6-ona e os grupos substituintes HP justificam uma maior estabilidade do complexo HP- $\beta$ CD:Cantin-6-ona. Portanto, acredita-se que o complexo HP- $\beta-C D$ :Cantin-6-ona seja mais eficiente no incremento da solubilidade da Cantin-6-ona.

\section{REFERENCIAS}

DAI, J. K. et al. Synthesis, in vitro antibacterial activities of a series of 3-N-substituted canthin-6-ones. Bioorganic and Medicinal Chemistry Letters, v. 26, n. 2, p. 580-583, 2016.

HAYNES, H. F.; NELSON, E. R.; PRICE, J. R. Alkaloids of the Australian Rutaceae: Pentaceras australis Hook. F. I. Isolation of the Alkaloids and Identification of Canthin-6-one. [s.l: s.n.]. v. 5 
KURKOV, S. V.; LOFTSSON, T. Cyclodextrins. International Journal of Pharmaceutics, v. 453, p. 167-180, 2013.

LOFTSSON, T.; DUCHÊNE, D. Cyclodextrins and their pharmaceutical applications. International journal of pharmaceutics, v. 329, n. 1-2, p. 1-11, fev. 2007.

RABELLO, 2016. Desenvolvimento e automação de metodologias in silico para o estudo de complexos de inclusão utilizados na inovação terapêutica. p. 104, 2016.

SAENGER, W. et al. Structures of the Common Cyclodextrins and Their Larger AnaloguesBeyond the Doughnut. Chemical Reviews, v. 98, n. 5, p. 1787-1802, jul. 1998.

SILVA, C. V. N. S. et al. Molecular modeling and cytotoxicity of diffractaic acid: HP- $\beta-$ $\mathrm{CD}$ inclusion complex encapsulated in microspheres. International Journal of Biological Macromolecules, v. 92, n. 5, p. 494-503, nov. 2016.

SORIANO-AGATÓN, F. et al. Extraction, Hemisynthesis, and Synthesis of Canthin-6one Analogues. Evaluation of Their Antifungal Activities. Journal of Natural Products, v. 68 , n. 11 , p. 1581-1587, nov. 2005.

TREIB, J. et al. An international view of hydroxyethyl starches. Intensive care medicine, v. 25, p. $258-68,1999$.

TREMMEL, T. et al. Canthin-4-ones as Novel Antibacterial Agents. Archiv der Pharmazie, v. 349, p. 710-723, 2016.

TROTT, O.; OLSON, A. J. AutoDock Vina: improving the speed and accuracy of docking with a new scoring function, efficient optimization, and multithreading. Journal of computational chemistry, v. 31, n. 2, p. 455-61, jan. 2010.

VIEIRA TORQUATO, H. F. et al. Canthin-6-one induces cell death, cell cycle arrest and differentiation in human myeloid leukemia cells. Biochimica et Biophysica Acta (BBA) - General Subjects, v. 1861, n. 4, p. 958-967, 2017.

WENZ, G. Cyclodextrins as Building Blocks for Supramolecular Structures and Functional Units. Angewandte Chemie International Edition, v. 33, p. 803-822, 1994.

XAVIER-JUNIOR, F. H. et al. Supramolecular interactions between $\beta$-lapachone with cyclodextrins studied using isothermal titration calorimetry and molecular modeling. Journal of Molecular Recognition, n. February, p. 1-10, 2017. 